\title{
Feedback Control Procedure for Energy Efficiency Optimization of Microwave-Heating Ovens
}

\section{Suggested abbreviated title: Calibration Procedure for Microwave Oven}

\author{
Juan Monzó-Cabrera $^{(1)}$, J.L. Pedreño-Molina ${ }^{(1)}$ and A. Toledo ${ }^{(2)}$ \\ (1) Departamento de Tecnologías de la Información y las Comunicaciones
}

${ }^{(2)}$ Departamento de Tecnología Electrónica

Universidad Politécnica de Cartagena, Cartagena (Spain)

Fax: +34 968325973

e-mail: Juan.Monzo@upct.es, Juan.PMolina@upct.es, Ana.Toledo@upct.es

\begin{abstract}
This paper presents an automatic calibration procedure that permits to industrial or domestic microwaves ovens to work at optimal energetic efficiency conditions. This method is based on a low-power iterative measurement of the microwave-oven scattering parameters for several sample locations to find the most efficient position by measuring energy reflections at feeding ports and the coupling between them for each sample position. These low-power measurements have been validated through high-power microwave-heating tests showing that, at the optimal estimated position, microwave absorption and therefore temperature increment is maximum. The method provides good matching levels independently of the sample geometry and permittivity without the need of any external matching device.
\end{abstract}

Key words - microwave heating, efficiency optimization, microwave oven, sample positioning control

\section{INTRODUCTION}

Traditional adaptation methods in microwave ovens are well known. They usually consist of external devices such as tuners, mobile short-circuits, irises and stubs that may provide adaptation between the microwave source and the final microwave receptor, i.e. sample, achieving low reflections back to the magnetron and maximum power transfer to the sample [1,2]. Common problems of all those methods are the high sensibility 
to the variation of the dielectric properties, and shape and dimensions of the sample to be heated. Other authors have shown that it is possible to achieve high power efficiencies by using dielectric casts around the sample $[3,4]$. However, these methods have not been experimentally tested yet, showing only simulated data. Other methods have tried to optimize the efficiency of microwave heating devices from an electromagnetic point of view [5]-[6], although these methods are very slow mainly due to the high computational cost for each iteration during the optimization loop. Other problems from the classical methods, including mechanical elements, are related to the use of irises and waveguide tuning screws acting as triple or quadruple stubs [1, 2], which need to be finely tuned to provide an adequate match to load and frequency conditions. The proposed method gives a solution to the optimization problem with the advantage of being independent of the dielectric or geometrical properties of the material, as well as the specific design of the oven.

On the other hand, several previous experimental tests have shown that for a multimode microwave cavity, microwave power efficiency varies according to frequency, dielectric properties, position inside the cavity and geometric characteristics of the heated material $[7,8]$. In fact, in that work it is shown that for several dielectric samples, the efficiency varies with the distance to the magnetron by following a non-linear curve. Actually, notable differences of energy efficiency values between the best and worst positions are reported.

In this work, a novel feedback control procedure to place the sample at the appropriate distance to the magnetron, in terms of optimal power efficiency, is presented and implemented by using an experimental platform consisting of a multimode microwave oven, an electro-mechanic positioning system and one control system. Different tasks carried out to implement and test this new matching method are presented here: a) Development of a computer-controlled acquisition system of the $S_{11}$ parameter at the feeding port based on the use of a network analyzer; b) Design and implementation of a synchronized mobile tracking system; c) Implementation of the motor controller; d) three-dimensional simulations for cavity mode estimation and e) Analysis of efficiency improvement and system reliability. 


\section{OPTIMIZATION METHODOLOGY}

It is possible to define the energetic behaviour of a microwave oven by means of the efficiency parameter $(\eta)$, which represents the relationship between the incident and reflected power at the feeding port. When only one feeding port is considered, the energy efficiency and the reflection coefficient are related by the next expression :

$$
\eta=1-\left|S_{11}\right|^{2}
$$

with $S_{11}$ being the complex reflection coefficient at the waveguide feeding port.

On the other hand, the microwave power absorbed in the sample can be related to the average temperature rise in the sample as explained in [1]. Equation (2) shows this relationship:

$$
P_{a b s}=P_{i n c} \cdot \eta=m_{\text {sample }} \cdot c_{p} \cdot \Delta T / t
$$

where $P_{a b s}(\mathrm{~W})$ is the absorbed microwave power, $P_{\text {inc }}(\mathrm{W})$ the incident microwave power at the feeding port, $m_{\text {sample }}(\mathrm{kg})$ is the sample mass, $c_{p}\left(\mathrm{~J} / \mathrm{kg}{ }^{\circ} \mathrm{C}\right)$ its specific heat, $\Delta T\left({ }^{\circ} \mathrm{C}\right)$ the temperature increment and $t(\mathrm{~s})$ the irradiation time. Therefore, $\eta$ will be maximum when the average temperature increment in the sample is maximum and a constant incident microwave power is used.

In this paper the efficiency changes, as the sample distance to the magnetron varies, have been studied. The efficiency must be measured for different sample locations, as Figure 1 shows. There $D_{\max }$ is the maximum distance to be covered by the sample, while $D_{\text {opt }}$ represents the sample position corresponding to maximum power efficiency. $D$ is a generic position inside the cavity. In the proposed methodology, the sample is initially placed at $D_{\max }$ position. Then the measurement and control systems generate the appropriate trajectory for the sample along the axis represented in Figure 1. For each new position $D$, efficiency is measured according to (1), so it can be considered as a function depending on sample position, $\eta$ $=\eta(D)$.

The low-power measurement procedure ends when the sample reaches the last position, $D_{\min }$, of the trajectory. Once the low-power efficiency measurement is finished and $D_{\text {opt }}$ is estimated, the sample can be placed in the optimal position and heating can be initiated by using a high-power magnetron. 


\section{MICROWAVE OVEN DESIGN}

Previously to the construction of the microwave oven prototype, an electromagnetic (EM) simulation has been carried out, in order to verify that enough modes were present in the Industrial, Scientific and Medical (ISM) band around $2.45 \mathrm{GHz}$. For this task, $\mathrm{CST}^{\mathrm{TM}}$ Microwave Studio has been used and the analyzed mechanical system is shown in Figure 2 . The cavity dimensions $\left(60 \times 60 \times 60 \mathrm{~cm}^{3}\right)$ were selected in order to allow the propagation of enough modes inside it. Rectangular geometry was used since most industrial ovens present this configuration. The positioning device consists of a cartload, which is slipped over two plain cylindrical axes. It is propelled by the rotation movement of a screwed cylindrical central axis with a rectangular prism to transform rotation into translation movement.

Figure 2 also shows that in order to couple the axis of the motor to the positioning system, a cylindrical waveguide, with a $1.3 \mathrm{~cm}$. diameter and $9 \mathrm{~cm}$. length, has been placed opposite the magnetron. With these dimensions, the main mode of this circular waveguide is at cut-off condition, which avoids modes escaping trough the guide and therefore any external radiation. Nylon $(\varepsilon=3.02-\mathrm{j} 0.036)$ has been employed for the axis and the cartload manufacturing $\left(10 \times 12 \times 1 \mathrm{~cm}^{3}\right)$, because it can be easily mechanized, while stainless steel has been selected for the cavity walls.

Figure 3 shows the computed behavior of $\left|\mathrm{S}_{11}\right|$ between 2.4 and $2.6 \mathrm{GHz}$ for the cavity, with the cartload placed at its central position without any sample. At least 15 resonance frequencies can be distinguished in this graphic at the 2.4-2.5 GHz bandwidth, indicating enough number of modes in the oven for the ISM band.

\section{EXPERIMENTAL PLATFORM}

The developed microwave prototype consists of a positioning system, the rectangular-section cavity, a Rohde $\&$ Swartz Vector Network Analyzer (VNA) for $S_{11}$ measurement, a high-power microwave source (magnetron), one external thermographic camera and the interface to control the sample trajectory as well as 
the motor activation and $S_{11}$ acquisition. The software interface also permits to place the sample in the most appropriate position in terms of energy efficiency.

The thermographic sensor has been incorporated to the prototype in order to verify the correspondence between curves obtained whit low power irradiation (for $S_{11}$ parameter measurements) and those obtained when the magnetron is employed (high-power irradiation). In this case, the average temperature of the thermographic image of the sample has been taken into account as efficiency indicator. This parameter has been estimated by averaging the values for the pixels corresponding to the thermal image of the sample.

The positioning system is composed of the motor device and the nylon structural elements. The irradiation time was set around $60 \mathrm{~s}$ and maximum power was limited to $600 \mathrm{~W}$ to avoid sample overheating. In order to avoid vibrations during sample displacement, a flexible connection has been incorporated at the end of the central axis. With respect to the actuator system, an external DC MAXON 1444328 Swiss Mode $\mathrm{T}_{11}$ stepper motor has been employed, establishing a serial communication between the hardware controller and the computer. The stepper motor incorporates one encoder to provide the position at any instant. The relationship between encoder and position is employed to control the cartload inside the cavity. The screwed axis transforms the rotation movement into linear movement for the sample, with a relationship of $5.7 \times 10^{-4}$ $\mathrm{mm} / \mathrm{step}$ and a maximum linear velocity of $11.7 \mathrm{~mm} / \mathrm{s}$. Each complete turn of the axis is equivalent to $2 \mathrm{~mm}$ longitudinal sample movement.

A metallic grid has been placed at the top of the cavity in order to allow the capture of thermal images while avoiding microwave leakage. The average temperature of the thermal images obtained from the camera provides an estimation of the absorbed power for the sample, as described in $[1,2]$ and equation (2). The use of the metallic grid distorts the measured sample temperature. In order to compensate this attenuation, the relationship between the average temperature and the temperature, which is measured with the effect of the grid, has been computed from experimental tests. Finally, the average temperature is employed to measure 
the range of the efficiency, in terms of temperature increments between any position and the optimal position, for each material.

For the low-power calibration phase of the proposed methodology, the VNA has been employed to measure the $S_{11}$ parameter. This low-power phase consists of measuring the $S_{11}$ parameter for different sample positions which leads to the estimation of the minimum value for $\left|S_{11}\right|$. This minimum value will be the optimal position for the calibration procedure. Because the first level calibration is developed with power levels under $1 \mathrm{~W}$, the increments of temperature at the sample are located at the beginning of the heating curve, where low powers correspond to no significant variation of the temperature. Due to this, the calibration procedure with low-power avoids to consider the changes of material dielectric properties when increasing the temperature. Therefore, the calibration procedure with low-power avoids to consider the changes of material dielectric properties when increasing the temperature. For the second phase, the network analyzer is substituted by a commercial SAMSUNG OM75S magnetron. Both low and high-power procedures used the same waveguide launcher in order to be able to reproduce the same experimental conditions. Figure 4 shows the developed prototype while Figure 5 shows the cavity containing the positioning system and one small polyester sample. The experimental tests used 398 different positions (from $10 \mathrm{~cm}$ to $50 \mathrm{~cm}$ ) in order to properly characterize the resonances of the system. The materials employed in the experimentation were wood, polyester and epoxy resins and methacrylate, as described in Table 1.

\section{RESULTS}

In this section experimental results are shown, in order to verify two main aspects: the existence of an optimal position for maximum energy efficiency and efficiency improvement with different samples when they are placed at the optimal estimated position.

\section{A. Maximum Efficiency Determination}


Two examples of the obtained results can be observed in Figures 6 and 7. Figure 6 shows the evolution of $\left|S_{11}\right|$ with respect to the distance to the magnetron for the rectangular (C) and cylindrical (D) polyester samples at $2.45 \mathrm{GHz}$, while Figure 7 shows the same results but comparing the epoxy and rectangular polyester samples (samples B and C respectively). From these curves, it is possible to conclude that there is at least one point within the microwave cavity at which the $\left|S_{11}\right|$ is minimum and therefore power efficiency is maximized. Indeed, it can also be observed that small variations around the optimal position can produce significant variations of $\left|S_{11}\right|$.

Moreover, one can be appreciate in Figure 6 that at long distances $\left|S_{11}\right|$ is not different for different sample shapes, but at short distances differences are bigger. This may be explained by the fact that for short distances from sample to feeding port there is more coupling between these two elements whereas for long distances the effect of the whole cavity is more important over the final result.

Both curves in Figure 7 show a very similar behaviour although a shift in distance can be appreciated. This is a very typical behaviour of microwave resonant structures or cavities where small permittivity values variations lead to shifts in resonances or minimum values for $\left|S_{11}\right|$. Although these shifts are often observed in the frequency domain, this can be translated to distance since both frequency and distance are related through waveguide electrical length.

This implies that an important increment of the efficiency can be achieved by adequately relocating the position of the sample. On the other hand, it can also be observed that, for the studied samples, both sample shape and permittivity have a great influence on the power-efficiency optimal position.

\section{B. The High-Power process}

The second step of the proposed calibration methodology consists of sample irradiation at high power rates by using a magnetron as microwave source. To accomplish the maximum efficiency objective, the sample must be placed at the optimal position estimated from the low power procedure. Thus, it is necessary to verify that there is a clear correspondence between the obtained low-power optimal position and the optimal position when high-power is applied. Figure 8 shows the power efficiency as a function of distance obtained 
from $\left|S_{11}\right|$ measurements versus normalized averaged temperature increase obtained at high microwave power levels for sample C. In this case, since magnetron spectrum presented resonant frequency around $2.475 \mathrm{GHz}$, all low-power measurements were collected at this frequency.

From these data, it can be concluded that both methods estimate the best power efficiency position very closely. Differences in position may be due to magnetron frequency drifts, since load impedance changes during the irradiation procedure.

Finally, in order to evaluate the efficiency improvement obtained with this prototype and methodology, experiments at $600 \mathrm{~W}$ with samples A, B, C and D have been carried out. In this case, the samples were irradiated at the best and worst efficiency positions provided by the low power procedure and their averages temperatures recorded. The differences for the final average temperature between both positions have been calculated and the results are summarized in Table II, where $\Delta T_{a}=T_{\triangle P O s}-T_{O P}$, being $T_{O P}$ the temperature reached by the sample after the irradiation at the optimal position, and $T_{\triangle P o s}$ the temperature reached when the sample is displaced $\triangle P O$ s from the optimal point. It can be observed that small displacements on the sample position can lead to very different final average temperatures even for very short irradiation times (20

s). In fact, for sample A average temperature difference is around $54.7^{\circ} \mathrm{C}$ and for sample B it is around 39 ${ }^{\circ} \mathrm{C}$.

\section{CONCLUSIONS}

This paper presents the characteristics, possibilities and applications of a novel calibration methodology which allows improving the efficiency of microwave ovens without external devices. This calibration procedure uses information of measured values of $S_{11}$ when the sample is irradiated at different positions inside the cavity.

The proposed methodology has been experimentally verified at high power levels by using thermographic 
measurements. Therefore, it has been proved that placing the samples at the specific position within the microwave cavity where $S_{11}$ is a minimum has provided the best sample average temperature increment. 


\section{REFERENCES}

[1] A.C. Metaxas, R.J. Meredith, Industrial Microwave Heating, Peter Peregrinus Ltd., London, 1983

[2] R.J. Meredith. Engineers’ Handbook of Industrial Microwave Heating, IEE, London, 1988

[3] J. Monzó-Cabrera, A. Díaz-Morcillo, J.L. Pedreño-Molina, D. Sánchez-Hernández, A New Method for Load matching in multimode-microwave heating, applicators based on the use of dielectric layer superposition, Microwave and Optical Technology Letters, 2004, Vol. 40, No 4, pp. 318-322

[4] J. Monzó-Cabrera, J. Escalante, A. Díaz-Morcillo, A. Martínez-González, D. Sánchez-Hernández, Load matching in multimode microwave-heating applicators based on the use of dielectric-layer moulding with commercial materials, Microwave and Optical Technology Letters, 2004,Vol. 41, pp. 414-417

[5] E. Murphy and V.V Yakovlev, "FDTD-backed RBF network technique for efficiency optimization of microwave structures", Proc. 9th AMPERE Conference on Microwave and High Frequency Heating (Loughborough, U.K., September 2003), pp. 197-200, 2003.

[6] J. Monzó-Cabrera, J. Escalante, A. Díaz-Morcillo, A. Martínez-González and D. Sánchez-Hernández, "Load Matching in Multimode Microwave-Heating Applicators Based on the Use of Dielectric-Layer with Commercial Materials", Microw. Opt. Technol. Lett., Vol. 41, no. 5, pp. 414-417, 2004.

[7] M.E. Requena-Pérez, J.L. Pedreño-Molina, J. Monzó-Cabrera, A. Díaz-Morcillo, Multimode Cavity

Efficiency Optimization by optimum load location: experimental approach, IEEE Transactions on Microwave Theory and Techniques, 2005, Vol. 53, pp. 2838-2845

[8] J.L. Pedreño-Molina, J. Monzó-Cabrera, M.E. Requena-Pérez, A. Díaz-Morcillo, A Novel Optimization Procedure to Improve the Efficiency of Microwaves Ovens in Heating Processes, $40^{\text {th }}$ Annual Microwave Power Symposium, Boston (USA), 2006 


\section{FIGURE CAPTIONS}

Figure 1. Graphic representation of a microwave oven to compute the optimal distance for maximum efficiency.

Figure 2. 3D cavity design, including the positioning device

Figure 3. Computed $\left|S_{11}\right|$ variation from 2.4 to $2.6 \mathrm{GHz}$, without sample inside the oven

Figure 4. Experimental platform

Figure 5. Polyester sample on the positioning system

Figure 6. $\left|S_{11}\right|$ variation versus sample position for several geometry polyester samples (samples C and D) at $2.45 \mathrm{GHz}$.

Figure 7. $\left|S_{11}\right|$ variation versus sample position for several materials (polyester and epoxy resins) with the same dimensions (samples B and C) at $2.45 \mathrm{GHz}$.

Figure 8. $\left|S_{11}\right|$ behaviour and normalized temperature increment for sample C, at low-power and high-power levels at $2.475 \mathrm{GHz}$. 


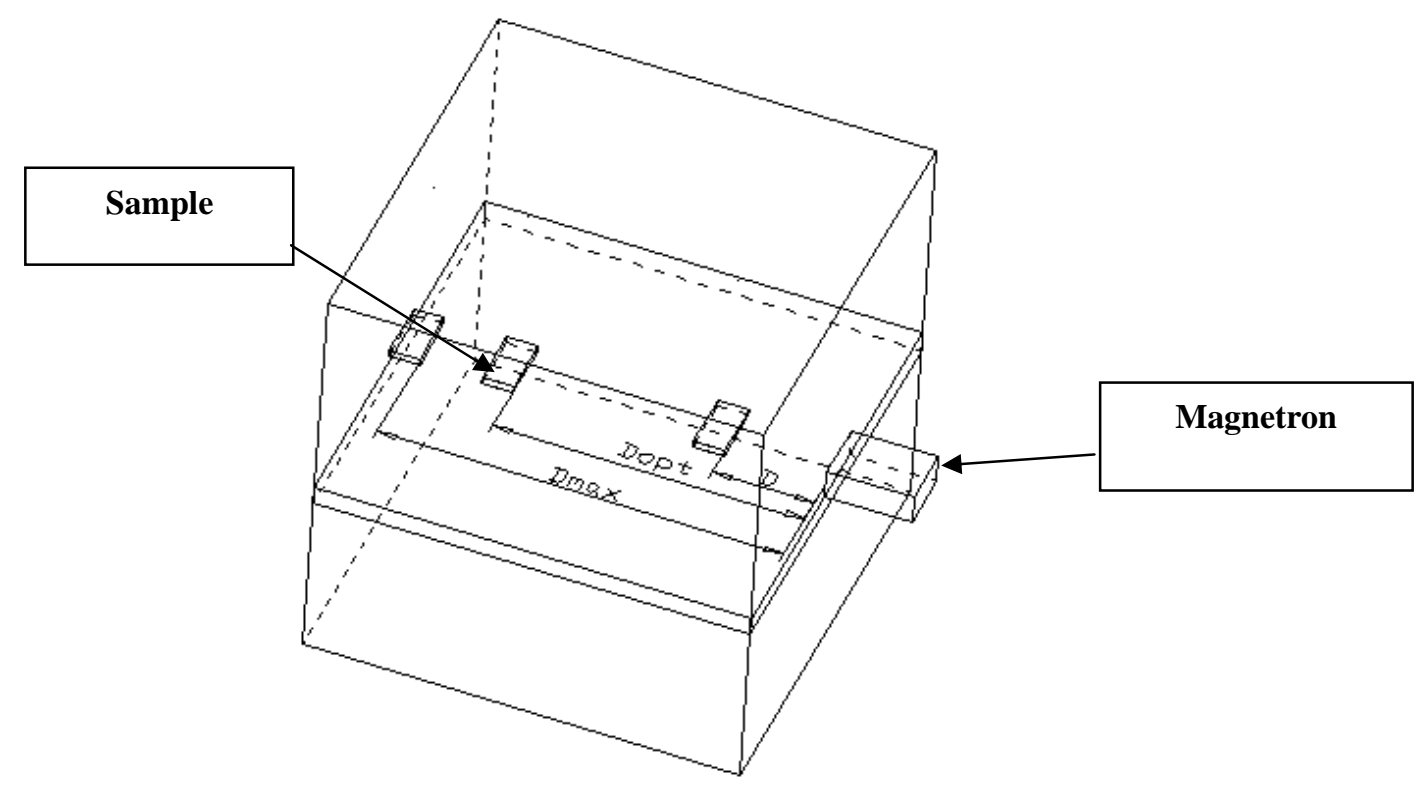

(Fig. 1) 


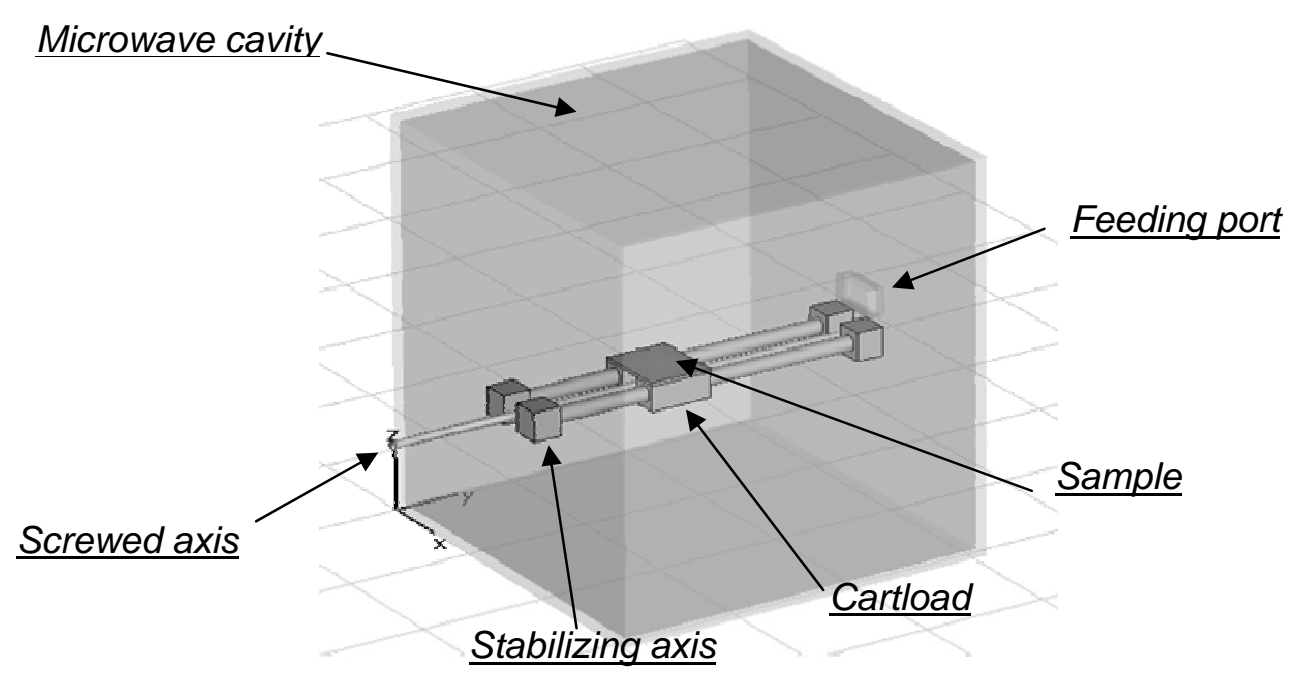

(Fig. 2) 


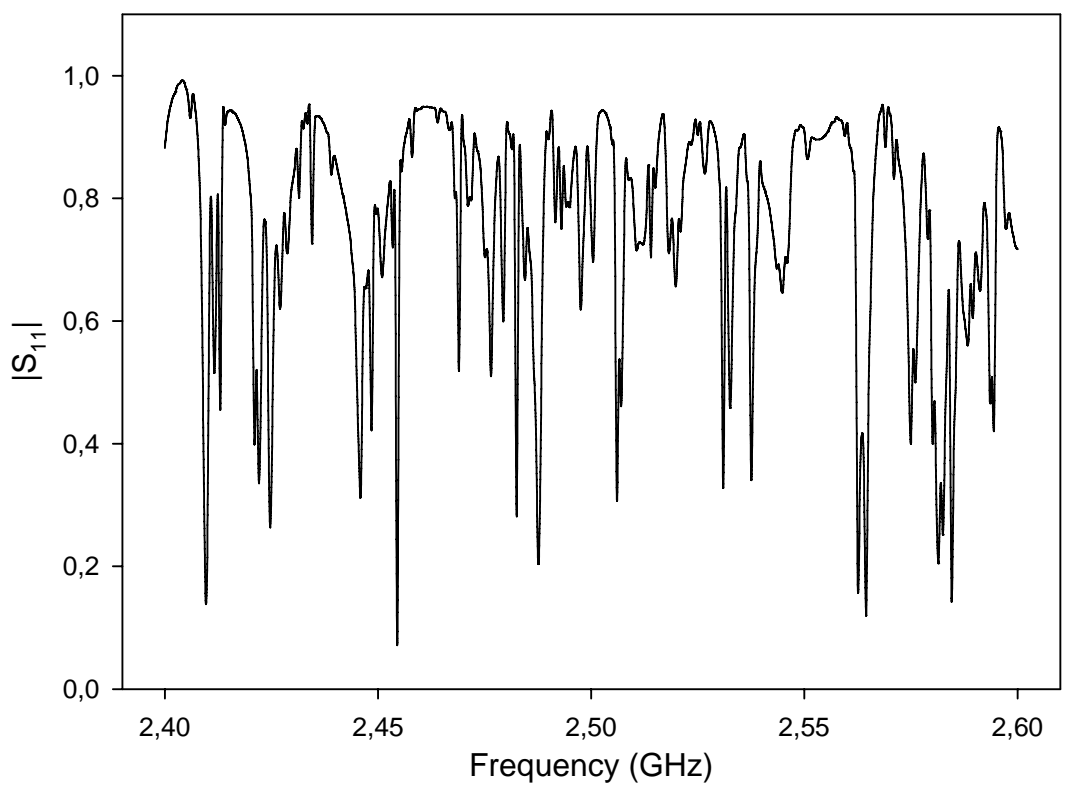

(Fig. 3) 


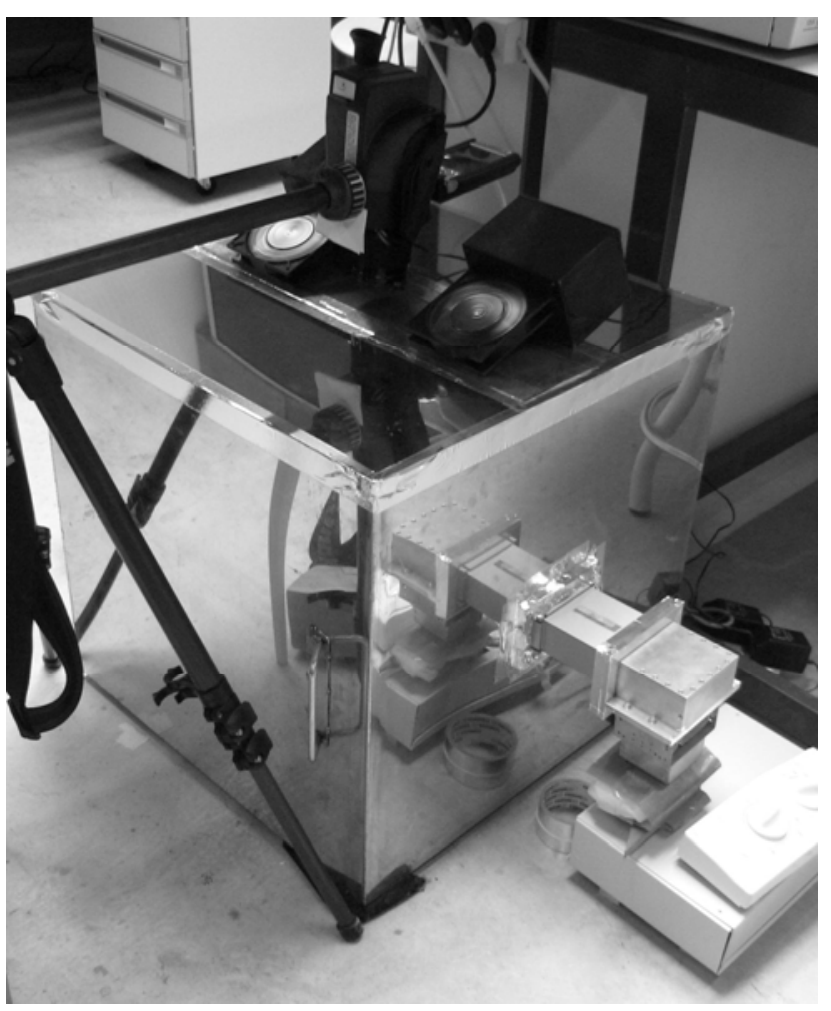

(Fig. 4) 


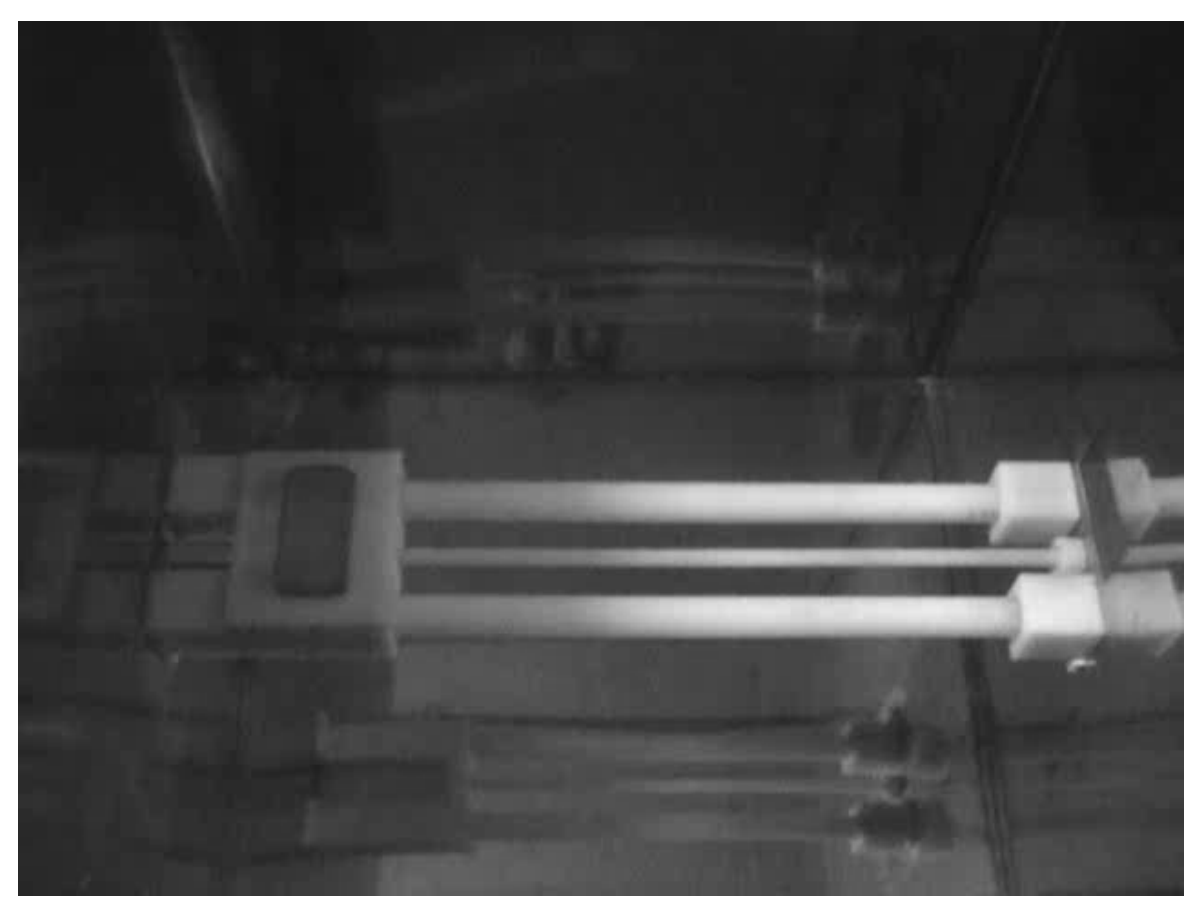

(Fig. 5) 


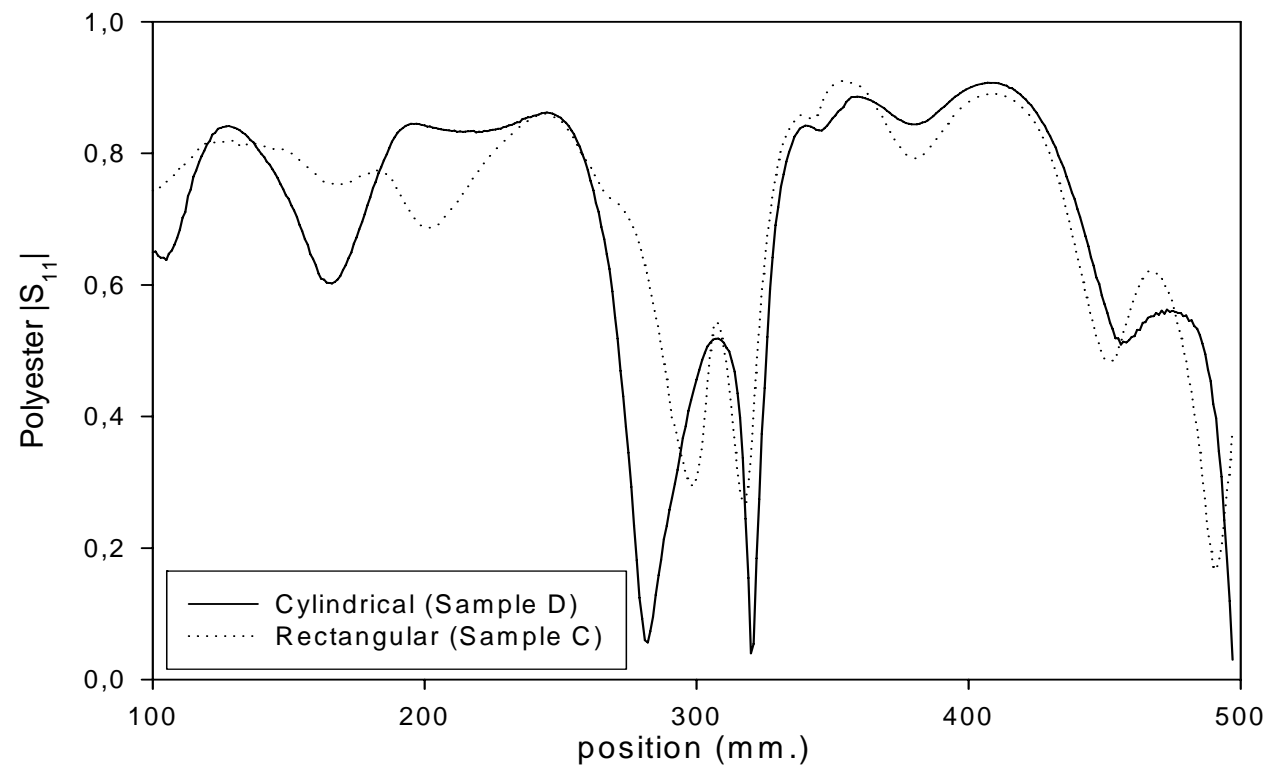

(Fig. 6) 


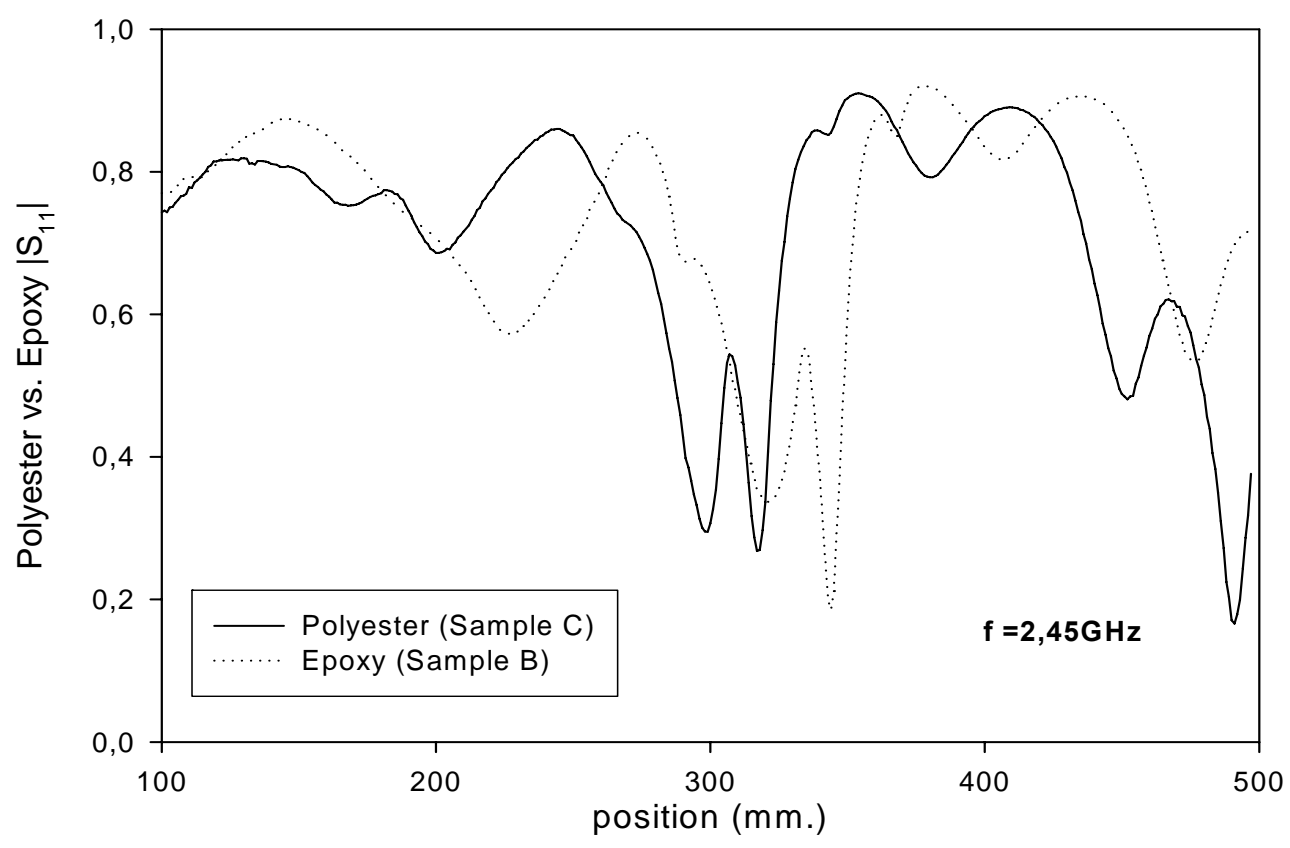

(Fig. 7) 


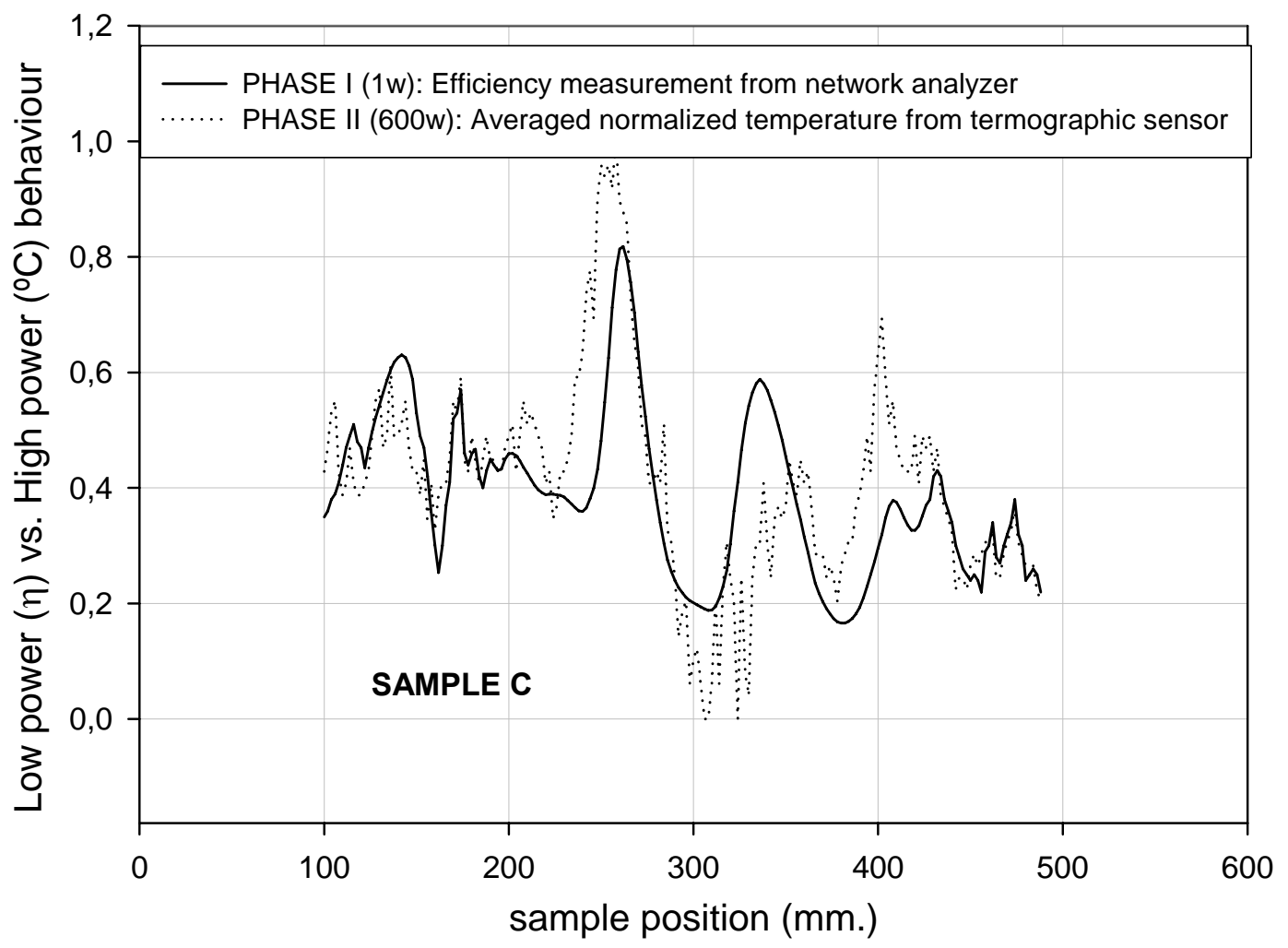

(Fig. 8) 


\section{TABLE CAPTIONS}

Table I. Technical details of the employed samples

Table II. Analysis of efficiency improvement 


\begin{tabular}{clrc}
\hline & \multicolumn{1}{c}{ Sample } & Dimensions (cm) & $\begin{array}{c}\text { Permittivity } \\
\mathbf{( 2 . 4 5 G H z )}\end{array}$ \\
\hline A & Wood & $4,0 \times 5,2 \times 1,0$ & $1,9900-j 0,1900$ \\
B & Epoxy Resin & $4,3 \times 8,6 \times 1,0$ & $4,5488-j 1,4986$ \\
C & $\begin{array}{l}\text { Polyester } \\
\text { (rectangular) }\end{array}$ & $4,3 \times 8,6 \times 1,0$ & $4,3825-j 0,4984$ \\
D & Polyester (cylindrical) & $\begin{array}{r}\text { Radius }=5,5 \\
\text { Thickness }=2,5\end{array}$ & $4,3825-j 0,4984$ \\
\hline
\end{tabular}

(Table 1) 


\begin{tabular}{ccccc}
\hline Sample & $\begin{array}{c}\Delta \mathrm{T}_{\mathrm{a}} \\
\left({ }^{\circ} \mathbf{C}\right)\end{array}$ & $\begin{array}{c}\Delta \text { Pos } \\
(\mathbf{m m} .)\end{array}$ & $\begin{array}{c}\text { Irradiation } \\
\text { Time }(\mathbf{s e c})\end{array}$ & $\begin{array}{c}\text { Optimal position } \\
(\mathbf{m m} .)\end{array}$ \\
\hline A & -54.7 & 48 & 20 & 252 \\
B & -39.1 & 72 & 60 & 248 \\
C & -37.1 & 72 & 60 & 252 \\
D & -26.7 & 68 & 60 & 268 \\
\hline
\end{tabular}

(Table 2) 